\title{
SOCIEDAD DE LA INFORMACIÓN EN EXTREMADURA: HACIA UN FUTURO MÁS LIBRE Y SOLIDARIO
}

\author{
Luis Miguel Cabezas Granado \\ Centro de Fomento de Nuevas Tecnologías (Junta de Extremadura)
}

\section{INTRODUCCIÓN}

El proyecto estratégico de Extremadura de acceso a la Sociedad de la Información está apoyado en los principios irrenunciables de la conectividad y la alfabetización tecnológica, y se marca como objetivo mejorar la calidad de vida de los extremeños desde la igualdad y la libertad.

Con este motivo, en la Región se han desarrollado una serie de acciones que, por una parte, la han dotado de una potente infraestructura de comunicaciones, la intranet regional, capaz de conectar, por un sistema de banda ancha, más de 1400 puntos distribuidos por los 383 municipios que integran la Comunidad Autónoma; y, por otra, se han puesto en marcha programas con los que alcanzar objetivos tanto de carácter educativo como socioeconómico.

Así mismo, se tenía claro que el pilar más importante para esta estrategia es la educación y que las Tecnologías de la Información y la Comunicación podían contribuir de manera decisiva a la mejora de la calidad de la enseñanza. De esta forma se planeó y comenzó a ejecutarse el despliegue de la Red Tecnológica Educativa (RTE), que está dotando de un ordenador por cada dos alumnos a los centros de educación secundaria. También se diseñó un Plan de Alfabetización Tecnológica (PAT) que no olvidase las necesidades de sectores de la población que llegaban tarde a este nuevo mundo tecnológico. Los 33 Nuevos Centros del Conocimiento repartidos por el territorio extremeño, y especialmente por localidades del ámbito rural, tienen esa finalidad. Finalmente, para terminar de cubrir todos los ámbitos de la sociedad, está Vivernet, que cumple la función de vivero de empresas 
de la nueva era digital, o el Centro de Fomento de Nuevas Iniciativas, encargado de ir analizando y dirigiendo la estrategia extremeña de Sociedad de la Información según sugieran las cambiantes circunstancias de cada momento.

En este contexto, y como fondo transversal, está enmarcado el Proyecto GNU/LinEx (programas libres, Free Software), nacido como respuesta a la necesidad de evitar que el éxito de todo el programa pudiera depender de factores externos que, como el software propietario, escapan a cualquier tipo de control público.

\section{CONTEXTO. CARACTERÍSTICAS SOCIOECONÓMICAS DE EXTREMADURA}

Extremadura tiene una extensión de $41634 \mathrm{~km}^{2}$ y una población de 1073574 habitantes, lo que supone una densidad de población de 25,78 habitantes por $\mathrm{km}^{2}$. El territorio de la región supone el 8,3\% del total de España y la población el 2,6\% del total de España. La población se distribuye en 383 municipios, y sólo el de Badajoz (136 319 habitantes) supera los 100000 habitantes. El $57 \%$ de los extremeños residen en municipios de menos de 10000 habitantes. El segundo municipio en número de habitantes es Cáceres, con 82 034, y el tercero, la capital autonómica, Mérida, con 51056.

La economía extremeña presenta una favorable evolución en los últimos años, siendo la comunidad autónoma española con mayor convergencia relativa con la Unión Europea en el periodo 1985-1999. Extremadura ha sabido aprovechar los Fondos de Cohesión de la Unión Europea para articular diversos proyectos en lo educativo, en lo social y en el sector empresarial. Proyectos que, bajo la premisa de incorporar la región a la revolución de las nuevas tecnologías y el conocimiento, están propiciando un desarrollo de la misma en condiciones de igualdad y libertad, y poniéndola en disposición de poder afrontar con garantías cuantos cambios depare de aquí en adelante la revolución del conocimiento. 


\section{LA ESTRATEGIA GLOBAL DE SOCIEDAD DE LA INFORMACIÓN DE EXTREMADURA}

Fue en 1998 cuando el Presidente de Extremadura, Juan Carlos Rodríguez Ibarra, en el Debate sobre Política General, anunció que los esfuerzos del Gobierno extremeño en las próximas legislaturas se iban a dirigir a conseguir que la Región estuviera a la cabeza de la revolución tecnológica conocida como la Sociedad de la Información, una revolución que tendría que afectar a toda la población y que estaría basada en los principios de la conectividad y la alfabetización tecnológica, con el fin de mejorar la calidad de vida de los ciudadanos.

Habíamos perdido la oportunidad de estar en las revoluciones industriales y ahora se presentaba una oportunidad única para liderar esta revolución. La Sociedad del Conocimiento, cuya materia prima no es el carbón, ni el petróleo, sino la información y las redes informáticas como medios de transmisión, pone en el mismo lugar, con las mismas oportunidades de éxito, a cualquier persona, independientemente del lugar físico en que se encuentre.

La apuesta era ambiciosa y por ello se pusieron en marcha desde la Consejería de Educación, Ciencia y Tecnología una serie de medidas para dotar a la Región tanto de las infraestructuras de telecomunicaciones como de los programas necesarios para poder alcanzar los objetivos tanto de carácter educativo como socioeconómicos.

\subsection{Marco tecnológico: Intranet de Extremadura}

La intranet de Extremadura comenzó a desplegarse en 1999 y tenía como objetivo dotar de acceso a banda ancha (2 Mb reales) a todas las poblaciones de la Región a través de los centros educativos, sanitarios o administrativos de la Junta de Extremadura. A finales del año 2001 la intranet estaba completamente desplegada, con 1800 puntos de conexión, y todas las poblaciones de Extremadura, cerca de 400, tienen acceso a banda ancha y salida a internet. 
Técnicamente, la intranet se estructura en un anillo de fibra óptica que une a todos los centros administrativos con el punto único de acceso, que se encuentra en el Centro de Proceso de Datos de Mérida. En los centros a los que no llega la fibra se utiliza la tecnología de radioenlace. De esta manera, por toda la geografía de la Región existe una red de antenas de transmisión y recepción de datos que conectan el punto único de acceso con las diferentes poblaciones. De la misma forma, se está utilizando la tecnología WIFI para interconectar edificios que están separados físicamente, como es el caso de algunos centros educativos en determinadas poblaciones, en las que la conexión a la intranet llega a un edificio, pero dispone de otros dependientes del mismo centro.

En un futuro, existe la posibilidad de utilizar los remanentes de la intranet para ofrecer servicios de conexión a internet a la población en aquellos lugares a los que no lleguen tecnologías como la ADSL. Mediante esta fórmula se utilizaría tecnología WIFI para distribuir la señal a los distintos puntos de la población.

\subsection{Marco estratégico}

Al mismo tiempo que comenzaba a prepararse el diseño de la intranet regional, se pusieron en marcha una serie de programas dirigidos a los distintos ámbitos de la sociedad extremeña, orientados principalmente a la alfabetización tecnológica de la población, al fomento de las oportunidades de negocio basadas en nuevas tecnologías, a la promoción de la Sociedad de la Información y de las nuevas tecnologías y a la mejora del sistema educativo.

\subsubsection{Plan de Alfabetización Tecnológica}

El Plan de Alfabetización Tecnológica (PAT) fue el primero que puso en marcha el Gobierno regional a finales de 1999 con el fin de asegurar el acceso universal de todos los ciudadanos a las Tecnologías de la información. Este proceso de alfabetización tecnológica se estructura a través de una serie de centros denominados Nuevos Centros del Conocimiento. Actualmente existen 33 Nuevos Centros del Conocimiento (NCC) repartidos por toda la 
Región, principalmente en las zonas rurales alejadas de las grandes ciudades y en las zonas urbanas más desfavorecidas.

En estos Centros se ofrece a los usuarios, principalmente población adulta, formación en el uso de las nuevas tecnologías según sus necesidades, generalmente en la familiarización y uso de las herramientas informáticas. Cabe destacar el elevado número de personas mayores que aquí han aprendido a utilizar el ordenador y a navegar o utilizar el correo electrónico.

Cada NCC dispone de un técnico informático, un dinamizador social, ocho ordenadores con conexión a internet y los periféricos más usuales (escáner, impresora, cámara digital, etc.), y desde la presentación de GNU/LinEx todos estos centros usan este software libre sin que hasta el momento haya habido problema reseñable alguno. Desde que está funcionando GNU/LinEx han pasado más de 50000 personas por los eventos realizados en los NCC y el sistema ha tenido una buena acogida por parte de los usuarios.

\subsubsection{Vivernet}

Vivernet, Viveros de Empresa en la Nueva Era, nació a mediados del año 2000 con el fin de apoyar a los emprendedores de negocios de base tecnológica. A través de un espacio físico en los viveros que existen en Badajoz y Cáceres, la nueva empresa acogida en Vivernet recibe durante una media de 15 meses asesoramiento legal y económico, además de formación en diversas materias tanto empresariales como tecnológicas.

Desde Vivernet se viene impulsando la utilización de GNU/LinEx y el desarrollo de software libre entre las PYMES de la Región, a través de cursos de formación y jornadas sobre posibilidades de negocio de este tipo de software.

\subsubsection{Centro de Fomento de Nuevas Iniciativas}

El Centro de Fomento de Nuevas Iniciativas es quizás el más desconocido ya que trabaja de una forma más interna para la Administración; es el encargado de analizar y definir estrategias en 
materia de Sociedad de la Información, así como de fomentar el uso y desarrollo de las tecnologías de la Información en los distintos ámbitos de la sociedad. El programa eExtremadura, ejecutado por el Centro en los últimos dos años, es una acción financiada con fondos europeos que trata de unir a distintos agentes regionales (sindicatos, empresas, ayuntamientos, universidad) para desarrollar proyectos en los que se empleen las TIC. En la segunda convocatoria, después de la presentación de GNU/LinEx, de los más de 400 proyectos presentados, más de 50 mencionaban el uso de GNU/LinEx o software libre para su ejecución.

En la actualidad, el Centro de Fomento de Nuevas Iniciativas es el que coordina el desarrollo técnico del proyecto GNU/LinEx, en el que intervienen la Consejería de Educación, Ciencia y Tecnología, los Nuevos Centros del Conocimiento, Vivernet y Fundecyt.

\subsubsection{Red Tecnológica Educativa}

La Red Tecnológica Educativa de Extremadura es el proyecto más emblemático de la Consejería de Educación, Ciencia y Tecnología. Su objetivo es incorporar la Sociedad del Conocimiento al Sistema Educativo, y para ello se pusieron en marcha acciones como la formación en el uso de las TIC a los docentes o el fomento de la creación de contenidos compartidos a través de la red (www.extremadurasi.org).

Pero quizás la acción más ambiciosa era pasar del concepto del «aula de ordenadores» al de «el ordenador en el aula», estableciendo una ratio de un ordenador por cada dos alumnos en los centros de secundaria, lo que influyó incluso en la arquitectura de los propios centros de nueva construcción: las aulas son más grandes y en la estructura del edificio están incluidos el cableado necesario para la red de datos y de electricidad de los ordenadores. Dentro de las aulas se ha instalado un nuevo tipo de mesa mucho más grande para poder albergar al ordenador y tener espacio para el trabajo habitual de los alumnos. En los centros que ya estaban construidos se ha diseñado una mesa un poco más pequeña. 
Es en este punto donde surge la necesidad de encontrar una solución para el software que debía incluirse en los ordenadores. El software propietario había dejado de ser válido y se necesitaba una alternativa que hiciera viable la instalación de 80000 máquinas. Esa alternativa era el software libre.

4 EL PROYECTO GNU/LINEX

A finales del año 2001 teníamos la intranet completamente operativa, los proyectos de la Dirección General de Sociedad de la Información se habían consolidado y, además, estaba finalizando la construcción de los 14 nuevos centros educativos en los que la ratio era de un ordenador por cada dos alumnos y como media tenían unos 400 equipos por centro.

Cada vez estábamos más arriba y se nos presentaba entonces un gran problema. Por una parte, en los proyectos (como proyectos tecnológicos) teníamos una gran dependencia del software, que además solía ser siempre de la misma gran empresa. Teníamos que hacer frente al pago de licencias para un número de máquinas muy respetable y las veces que habíamos tenido que renovar licencias, de paso, habíamos tenido que actualizar el hardware. Por otra parte, y quizás la más importante, en el sistema educativo había que instalar 80000 máquinas, las cuales además de sistema operativo tenían que contar con software de productividad.

Salta a la vista que la cantidad económica que había que desembolsar para hacer frente a tal cantidad de licencias de software era prácticamente inabarcable y mucho menos la renovación periódica de licencias cada vez que se actualicen los productos. Se hacía necesaria entonces una solución alternativa al software propietario, y la única solución posible era el software libre.

En el entorno de servidores, veníamos utilizando desde hacía tiempo software libre con unos resultados excelentes, pero todavía no habíamos probado una solución de software libre para un usuario final sin conocimientos de informática. Nuestra necesi- 
dad era contar con una solución de escritorio basada en software libre y orientada a un usuario con necesidades ofimáticas.

Para ello, sobre noviembre de 2001, nos pusimos en contacto con la empresa Ándago, que tenía experiencia de implantación de máquinas de escritorio con software libre en el Ministerio de Administraciones Públicas y, tras el visto bueno de los responsables de la Consejería, comenzaron a desarrollar la primera versión de GNU/LinEx.

\subsection{Sé legal, copia LinEx}

Con este eslogan se presentó GNU/LinEx el 17 de marzo de 2002, haciendo referencia a las ventajas que proporciona el software libre, como las de poder redistribuir los programas y el código con total libertad. Esta primera versión de Linex estaba basada en la distribución Debian Potato y como entorno gráfico incluía GNOME. Hay que reconocer que era una versión con muchas limitaciones pero que sirvió como punto de arranque: la instalación no hacía ninguna pregunta al usuario y ponía a su disposición un sistema productivo desde el principio.

Las primeras ventajas del software libre las comprobamos sólo un par de meses después. Dos profesores de Secundaria de la Junta de Extremadura habían desarrollado una nueva versión de Linex basándose en la que había desarrollado Ándago y mejorándola en todos los aspectos. Incorporamos a estos profesores al equipo técnico de GNU/LinEx de la Consejería de Educación, Ciencia y Tecnología y en septiembre de 2002 se presentó en el SIMO la versión GNU/LinEx 3.0 Woody, basada en Debian Woody y con GNOME 1.4 como entorno gráfico.

\subsection{Qué es GNU/LinEx}

Técnicamente, GNU/LinEx es una distribución de software libre basada en la distribución Debian GNU/Linux que incorpora una serie de programas y un entorno gráfico, GNOME, orientado a un usuario final no técnico. Debian es una distribución creada y mantenida por un numeroso grupo de programadores volunta- 
rios, investigadores y profesores sobre todo, de todo el mundo. La elección de Debian vino condicionada, por una parte, porque detrás de Debian no existe ninguna marca comercial que pueda desaparecer o cambiar sus políticas en un momento dado y, por otra, por los altos estándares de calidad que impone esta iniciativa a sus programas.

Pero la distribución Debian como tal no se ajustaba a nuestras necesidades, y el trabajo del equipo de desarrollo de GNU/LinEx ha consistido en adaptar Debian a las necesidades propias de la Junta de Extremadura. El primer trabajo que se hizo fue seleccionar el software que iba a aparecer en el único CD-ROM en el que se entrega GNU/LinEx: de los casi 9000 paquetes de software que componen Debian, se han seleccionado alrededor de 800, que son los que forman el núcleo de la distribución de GNU/LinEx. El siguiente trabajo que se realizó fue la creación de un instalador gráfico que simplificara el proceso de instalación al máximo y que no requiriera apenas la intervención del usuario. Finalmente, en el escritorio hemos añadido iconos que hacen referencia a la identidad cultural extremeña: por ejemplo, Espronceda es el editor de textos, Zurbarán es la herramienta de diseño gráfico o Guadalupe es el lector de correo electrónico. Hecho esto, obtuvimos un producto muy fácil de instalar y con un abanico de software de productividad que cubre prácticamente todas las necesidades de un usuario de oficina o del sistema educativo.

Actualmente, las características técnicas de GNU/Linex, versión 3.0rl+g2.2 son las siguientes:

- Kernel Linux 2.4.20

- Entorno gráfico GNOME 2.2

- Conjunto de aplicaciones libres GNU (GPL):

- Instalador gráfico (casi) automático

- Procesador de textos

- Hoja de cálculo

- Presentaciones

- Accesorios 
- Navegador web

- Correo electrónico

- Mensajería instantánea

- Gestión económica doméstica

— Tratamiento de imágenes

- VNC

- Y mucho más...

\subsection{GNU/LinEx en la red: www.linex.org}

De forma paralela a la presentación de GNU/LinEx se puso en marcha el portal www.linex.org, desde el que se canaliza toda la información que existe sobre el proyecto. Desde esta dirección se puede descargar GNU/LinEx desde diversos lugares y obtener soporte técnico a las dudas más usuales de los usuarios. También se puede acceder a la documentación, manuales y tutoriales que existen sobre GNU/LinEx.

Próximamente, se va a poner en marcha un servicio de asistencia técnica al que se va a poder acceder desde el portal. Los usuarios podrán formular sus consultas, que se responderán tanto en la propia web como en su correo electrónico.

\section{GNU/LINEX ES UN PROYECTO COLECTIVO}

A raíz de comenzar a trabajar en el proyecto de implantar software libre desde una administración pública, GNU/LinEx comenzó a tener una gran repercusión en la red. Era la primera vez que un gobierno hacía una apuesta tan clara por el uso de software libre y, ciertamente, en el entorno educativo Extremadura es pionera en el mundo al instalar un sistema operativo libre en las aulas. El proyecto GNU/LinEx ha sido portada de periódicos nacionales e internacionales, como el Washington Post o el International Herald Tribune, y ha atraído la atención de numerosos gobiernos y organizaciones, nacionales e internacionales, los cuales han expresado su intención de cooperar en el desarrollo de esta iniciativa. 
El primer acuerdo para cooperar en el desarrollo de software libre fue el protocolo suscrito entre la Junta de Extremadura y la Junta de Andalucía en marzo de 2003. Según este protocolo, Andalucía utilizaría los trabajos de Extremadura para desarrollar una distribución propia, denominada GNU/Guadalinex, que se presentará en septiembre de 2003. Para desarrollar el acuerdo, Andalucía y Extremadura han constituído un equipo técnico común para coordinar y compartir los avances que se realicen en cada comunidad autónoma.

Fuera de España, están previstos acuerdos de cooperación con el gobierno de Brasil y ha habido contactos con Argentina, Colombia y Perú.

Pero en el proyecto GNU/LinEx no solamente están participando gobiernos, también están participando asociaciones de usuarios y empresas. Tal es el caso de GULEX o SINUH, asociaciones extremeñas de usuarios de GNU/Linux, o Hispalinux, la asociación española de usuarios, sin las cuales GNU/LinEx no habría sido lo mismo.

El papel de las empresas en el proyecto ha sido quizás el más llamativo. Ya desde el primer momento observamos cómo otra de las ventajas que nos proporciona el uso de software libre es la dinamización del sector empresarial local relacionado sobre todo con el desarrollo de software que, aunque escaso, existe. Uno de los primeros frutos que ha dado la actividad de este sector ha sido el desarrollo de una serie de herramientas orientadas a la gestión económica de PYMES, contabilidad, nóminas, caja, etc. que serán incluidas en una versión de GNU/LinEx denominada GNU/LinEx edición para PYMES. Por supuesto, todo el software que se incluya en GNU/LinEx será liberado bajo la licencia GPL y el código fuente estará disponible en los servidores de www.linex.org.

Otras empresas que han entrado en contacto con el proyecto GNU/LinEx han sido OKI, que ha desarrollado para GNU/LinEx un instalador para todos sus modelos de impresoras; ACER, para incluir GNU/LinEx preinstalado en sus portátiles; 
Lambdaux Software, con los que existe un intercambio de tecnología entre GNU/LinEx y su distribución Lux que viene preinstalada en los ordenadores portátiles de la marca AIRIS. En definitiva, las grandes empresas entienden que hay una base importante de usuarios de software libre que representan una oportunidad de negocio.

\section{SOFTWARE LIBRE PARA LA ADMINISTRACIÓN PÚBLICA}

La experiencia que nos ha proporcionado el uso y la implantación de GNU/LinEx en el caso de Extremadura nos ha hecho llegar a una serie de conclusiones acerca de la aplicación del software libre en la Administración pública, dentro de las cuales hay muchas más ventajas que inconvenientes:

a) Gestión del dinero público. Las administraciones públicas, como tales, tienen un compromiso con sus ciudadanos para gestionar el dinero público mirando siempre por el beneficio de todos. Invirtiendo en software libre, la Administración devuelve a la sociedad parte de la inversión en forma de código fuente e invierte en I+D local al hacer uso de los servicios de las empresas de software de la Región. Además, al poder reutilizar el código fuente, se abaratan los costes de desarrollo de nuevas aplicaciones.

b) Licencia GPL vs. licencia propietaria. Al suscribir la compra de licencias de software propietario, la Administración acaba dependiendo de las políticas comerciales de, generalmente, un único proveedor, el cual puede cambiarlas en cualquier momento. Sin embargo, la licencia GPL hace posible la independencia del proveedor, y la Administración siempre podrá negociar con aquel que le proporcione un mejor servicio.

c) No se obliga a terceros a adquirir licencias de software propietario. Si en su comunicación habitual con los ciu- 
dadanos la Administración utiliza software propietario, está obligando a su vez a comprar software propietario a los ciudadanos. Sin embargo, si se utiliza software libre, el ciudadano tiene la oportunidad de elegir.

d) La Administración pública tiene también una responsabilidad como gran consumidor, pudiendo activar con su actuación los sectores tecnológicos basados en software libre.

En definitiva, el software libre fomenta una actitud tan positiva como es compartir el conocimiento y la cooperación a través de la Red, y plantea un modelo de negocio, basado en la prestación de servicios alrededor del Software, nuevo y viable, lo que hace que regiones como Extremadura puedan competir en igualdad de condiciones con otras regiones de todo el planeta. Viendo estas conclusiones, la pregunta a la que nos deben responder entonces es: ¿por qué seguir alquilando software propietario si existe el software libre? 